\title{
Herpes Zoster Brachial Plexopathy: Direct Steroid Injection
}

\author{
Coskun ARAZ1', Suna ASKIN', Cem YILMAZ² \\ ${ }^{1}$ Baskent University, Faculty of Medicine, Department of Anesthesiology, Ankara, Turkey \\ ${ }^{2}$ Baskent University, Faculty of Medicine, Department of Neurosurgery, Ankara, Turkey
}

\section{ABSTRACT}

\begin{abstract}
Herpes zoster (shingles) is a viral disease, characterized by painful skin eruptions and neuropathic sensory symptoms. Motor involvement and brachial plexus involvement in herpes zoster are rare conditions. Together with antiviral medication and pain therapy, palliative and supportive modalities take an important role in the treatment of herpes zoster. It is well documented in previous reports that oral or intravenous steroid administrations may be additive in management. In this case report, positive effects of direct steroid injection onto the brachial plexus via ultrasonography guidance in a patient with motor weakness due to herpes zoster involvement of brachial plexus is presented.
\end{abstract}

KEYWORDS: Herpes zoster, Brachial plexus, Steroid

\section{INTRODUCTION}

$\mathrm{H}$ erpes zoster $(\mathrm{HZ})$ is a viral disease, caused by an exclusively human alpha herpes virus and is characterized by segmental painful skin rash and sensory symptoms. The most common complication of $\mathrm{HZ}$ is post-herpetic neuralgia (PHN), a syndrome characterized by neuropathic pain and allodynia in the affected region, persisting for months or even years after the rash has healed. However, motor involvement is rare, and brachial plexus neuritis is also very rare $(2,3,6,7,10,11)$.

The main components of therapy of motor paresis or neuritis due to $\mathrm{HZ}$ are antiviral agents, pain therapy, and physiotherapy. Some studies have showed that the use of corticosteroids in combination with antiviral agents reduces pain and accelerates the healing process $(8,9,11,14)$. We present a case with motor involvement of shoulder due to $\mathrm{HZ}$ and its treatment with brachial plexus injection by interscalene approach with local anesthetic with low concentration and triamcinolone.

\section{CASE REPORT}

A previously healthy 69-year-old male patient was referred to our clinic with the history of progressive left arm weakness which started 2 weeks after $\mathrm{HZ}$ skin eruption affecting to the left $\mathrm{C} 4$ and $\mathrm{C} 6$ dermatomes. In the patient's history, the pain started with skin lesions and resolved at the end of the second weeks with oral acyclovir therapy. The patient did not define any pain at the admittance and he had no signs of cranial nerve or meningeal involvement. The skin lesions of $\mathrm{HZ}$ were extinguished and widely settled to periarticular region of the left shoulder, lateral surface of the left proximal arm and the left upper back. Motor strength examination of the left upper extremity revealed that motor power was $2 / 5$ on shoulder abduction, external rotation and elbow flexion, $3 / 5$ on elbow extension and $4 / 5$ on wrist and fingers. There were no pathological findings except for mild hypoesthesia at the periarticular area of left shoulder.

Patient's routine biochemical tests were within normal limits. Brain, diffusion-weighted brain and cervical magnetic resonance imaging (MRI) studies were normal at this time. Electrodiagnostic studies (EDS) revealed a brachial plexopathy affecting the upper, middle and lower trunks, but dominantly the upper trunk. The median, radial and ulnar nerves had slow conductivity whereas upper extremity muscles showed intensive degenerative properties in EDS. Moreover, brachial plexus MRI was performed and contrast media enhancement was observed at the left brachial plexus (Figures 1, 2). 


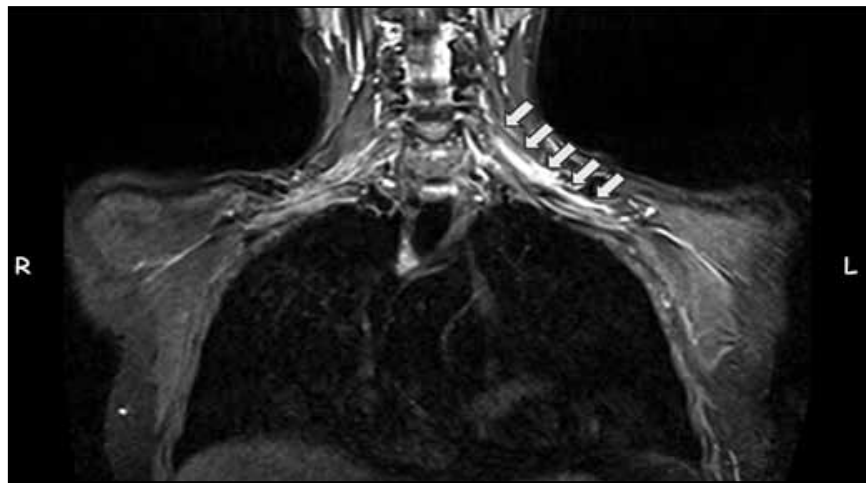

Figure 1: Brachial plexus MRI, contrast media enhancement was shown at left brachial plexus.

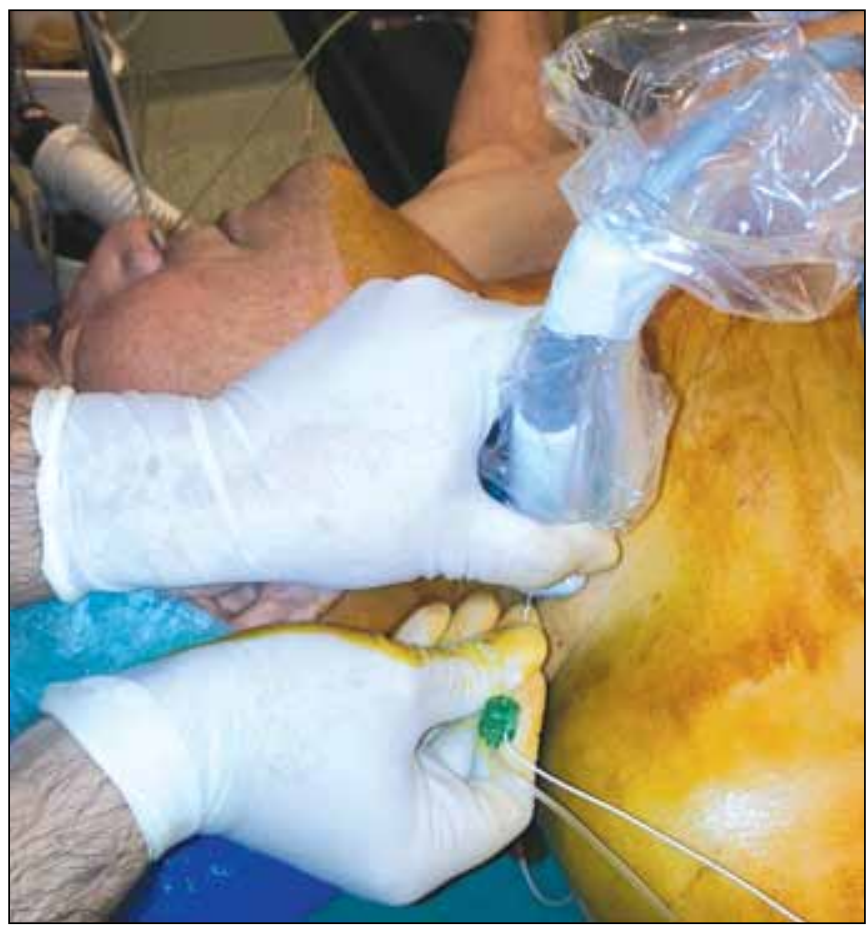

Figure 3: Demonstration of brachial plexus injection technique (Ultrasound guided interscalene approach).

For treatment, steroid was directly injected to the brachial plexus via interscalene approach under the guidance of ultrasonography (Figures 3, 4) with $40 \mathrm{mg}$ triamcinolone and $15 \mathrm{mg}$ bupivacaine in total of $15 \mathrm{~mL}$ volume, and physical therapy for 4 weeks was recommended. Only one week after injection, the patients' motor power was regained and weakness resolved within two months.

\section{DISCUSSION}

Herpes Zoster, better known as "shingles", is the reactivation of dormant varicella zoster virus in the dorsal root ganglia and is characterized by segmental rash, pain and sensory symptoms. The incidence and severity of $\mathrm{HZ}$ increase with age, immune system incompetency and stress $(1,3,7,10,11)$.

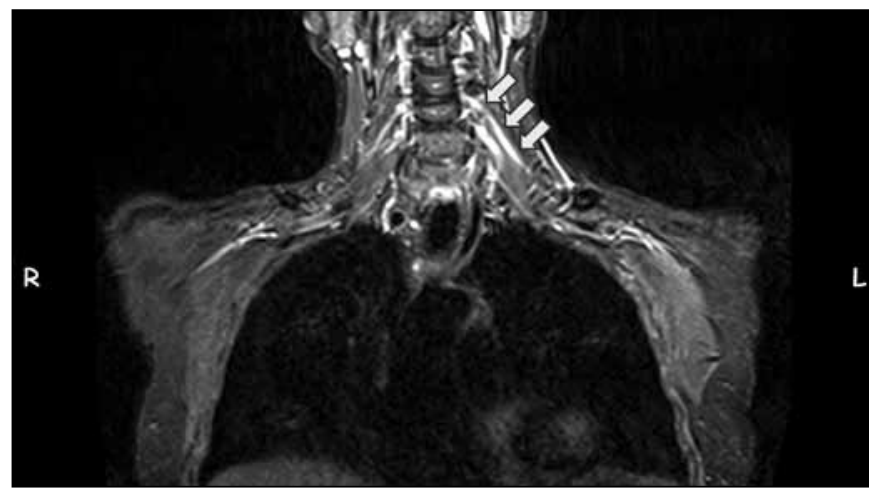

Figure 2: Brachial plexus MRI, contrast media enhancement was shown at left brachial plexus.

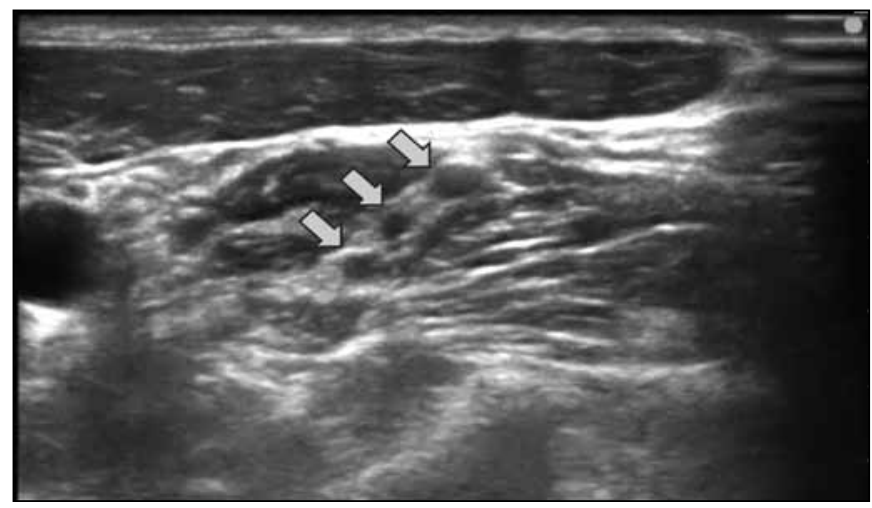

Figure 4: Ultrasound image of the brachial plexus in the interscalene groove (nerve roots marked by arrows).

Epidemiological studies have revealed motor involvement in $1-5 \%$ of patients with $\mathrm{HZ}(4,5,7)$. Brachial plexopathy is a very rare condition with an annual incidence of 1.64 cases per 100.000 persons (2). The associated motor neuropathy was probably due to an intense inflammatory demyelinating process and post-infectious immune-mediated motor root damage $(7,10)$. Fabian et al. reported in their post-mortem histopathological evaluation of herpes zoster neuritis that extensive lymphocytic infiltration and myelin breakdown occur in affected site (4).

Clinically, PHN is characterized by ongoing burning, tingling, shooting and mechanical allodynia. These symptoms usually spread to one or two dermatomes. Motor involvement is not common, and motor symptoms may occur 1 to 3 weeks before or after dermatological findings $(7,13)$. However, spreading of weakness or electrodiagnostic findings may not be in concordance with the involved dermatomes (1). Alshekhlee et al. comment in their report that, motor involvement ratio in $\mathrm{HZ}$ is higher than what is clinically detected. The low incidence of motor involvement of $\mathrm{HZ}$ is linked to difficult diagnosis of this pathological condition due to pain or subclinical conditions $(1,3)$. In this patient, dermatologic findings began to regress at the onset of the weakness and the motor deficit started to become predominant and the quality of his life gradually decreased. 
Since we were not able to observe the initial spread of the lesions, it was not possible to make a true comparison of motor and dermatological involvement. However, EDS revealed a brachial plexopathy affecting all, but dominantly the upper trunk of the brachial plexus.

Treatment of PHN is difficult, and a variety of treatments are offered without consensus about their effectiveness. Antiviral agents, corticosteroids and other pain management modalities are used $(8,9,11)$. Moreover, some interventional treatment modalities have also been reported including sympathetic blocks, epidural steroids, pulsed radiofrequency treatment or classic radiofrequency ablations $(11,12)$. But, there has been no report on an effective treatment for promoting functional recovery from zoster paresis. Physical, occupational or psychotherapy methods have been reported in some case reports or case series. In our patient, oral antiviral therapy started at the beginning of the eruptions and pain, after this therapy pain and skin lesions were healed. Two weeks after the initiation of the skin lesions the patient was admitted to the hospital with progressive left arm weakness, there was no complaint about pain this time. First examination and cervical MRI of the patient showed no possible pathology but EDS revealed a brachial plexopathy. Similar to the case of Ayoub et al. (2), brachial plexus MRI performed with these results showed inflammation of the brachial plexus.

Steroids were directly administered onto the brachial plexus for the treatment of inflammation. For this purpose, we used an ultrasound-guided anesthetic technique known as "interscalene blockade" which is used for brachial plexus block. The patient's weakness in the left arm almost completely resolved within 2 months. To the best of our knowledge, this is the first report of direct steroid injection to brachial plexus for $\mathrm{HZ}$ paresis. In our patient, progression of motor weakness disappeared in the first week with almost complete recovery at the end of second month. It has been previously reported that motor weakness could be resolved over a period of a few years (10). We attributed this rapid effect this expeditious effect of steroids to direct application in the inflammatory field whereby the inflammation in the affected site resolved immediately and functions rapidly returned.

\section{CONCLUSION}

Brachial plexus neuritis and motor paresis due to herpes zoster is a very rare condition, but it can limit daily life and can be disturbing for the patients. Since acute motor weakness may be related to many different reasons, the necessary radiological or electrodiagnostic tests should be performed. Additionally, if zoster pain or eruptions are included in the former history, motor involvement of $\mathrm{HZ}$ should be remembered. In such cases, direct steroid injection to the brachial plexus via different techniques should be kept in mind as an alternative treatment modality.

\section{REFERENCES}

1. Alshekhlee A, Tay E, Buczek M, Shakir ZA, Kakulas BA: Herpes zoster with motor involvement: Discordance between the distribution of skin rash and localization of peripheral nervous system dysfunction. J Clin Neuromuscul Dis 12: 153-157, 2011

2. Ayoub T, Raman V, Chowdhury M: Brachial neuritis caused by varicella-zoster diagnosed by changes in brachial plexus on MRI. J Neurol 257:1-4, 2010

3. Choi JY, Kang CH, Kim BJ, Park KW, Yu SW: Brachial plexopathy following herpes zoster infection: Two cases with MRI findings. J Neurol Sci 285:224-226, 2009

4. Fabian VA, Wood B, Crowley P, Kakulas BA: Herpes zoster brachial plexus neuritis. Clin Neuropathol 16(2):61-64, 1997

5. Forster M, Umnus A, Siebrecht D, Baron R, Wasner G: A case of pain, motor impairment, and swelling of the arm after acute herpes zoster infection. Pain 153: 2478-2481, 2012

6. Gopal KV, Sarvani D, Krishnam Raju PV, Rao GR, Venkateswarlu K: Herpes zoster motor neuropathy: A clinical and electrophysiological study. Indian J Dermatol Venereol Leprol 76: 569-571, 2010

7. Haanpaa M, Hakkinen V, Nurmikko T: Motor involvement in acute herpes zoster. Muscle Nerve 20:1433-1438, 1997

8. Han Y, Zhang J, Chen N, He L, Zhou M, Zhu C: Corticosteroids for preventing postherpetic neuralgia. Cochrane Database Syst Rev 3: CD005582, 2013

9. He L, Zhang D, Zhou M, Zhu C: Corticosteroids for preventing postherpetic neuralgia. Cochrane Database Syst Rev 1:CD005582, 2008

10. Ismail A, Rao DG, Sharrack B: Pure motor Herpes Zoster induced brachial plexopathy. J Neurol 256:1343-1345, 2009

11. Kawajiri S, Tani M, Noda K, Fujishima K, Hattori N, Okuma Y: Segmental zoster paresis of limbs: Report of three cases and review of literature. Neurologist 13:313-317, 2007

12. Lim SM, Park HL, Moon HY, Kang KH, Baek CH, Jung YH, Kim JY, Koo GH, Shin HY: Ultrasound-guided infraorbital nerve pulsed radiofrequency treatment for intractable postherpetic neuralgia - a case report. Korean J Pain 26:84-88, 2013

13. Wendling D, Langlois S, Lohse A, Toussirot E, Michel F: Herpes zoster sciatica with paresis preceding the skin lesions. Three case-reports. Joint Bone Spine 71:588-591, 2004

14. Whitley RJ, Weiss H, Gnann JW Jr, Tyring S, Mertz GJ, Pappas PG, Schleupner CJ, Hayden F, Wolf J, Soong SJ: Acyclovir with and without prednisone for the treatment of herpes zoster. A randomized, placebo-controlled trial. The National Institute of Allergy and Infectious Diseases Collaborative Antiviral Study Group. Ann Intern Med 125: 376-383, 1996 